\title{
COVID-19 and Sudden Cardiac Death in a Young Man with Myasthenia Gravis
}

\author{
Maysa Ghayyem ${ }^{1}$, Sara Haseli ${ }^{1}$, Shekoofeh Yaghmaei ${ }^{2 *}$ \\ ${ }^{1}$ Department of Internal Medicine, Faculty of Medicine, Tehran Medical Sciences, Islamic Azad University, Tehran, Iran \\ ${ }^{2}$ University of Fribourg, Fribourg, Switzerland
}

\begin{abstract}
Myasthenic patients are expected to develop more severe COVID-19 respiratory symptoms compared to the general population because of both respiratory muscle weakness and the possibility of an immunocompromised state related to immunotherapy. During a pandemic like COVID-19, such situation requires meticulous attention, as the entire population is potentially at risk of exposure. However, there is no data on best management for myasthenic patients with COVID infection.

Although pneumonia has been reported as the most common cause of death in COVID-19 patients, sudden cardiac death has also been described in the literature as a possible COVID-19 related mortality cause. Physicians should assess the patient's cardiac functionas precisely as possible, in addition to the respiratory condition, in order to prevent such incidents. Furthermore, as the cardiac system of myasthenic patients might be negatively influenced by their background, their cardiac system may be even more vulnerable during COVID-19, which should be noted bymedical teams. Reporting such cases can be helpful to develop protocols for treating myasthenic patients during the COVID-19 pandemic.

Here, we present a 37-year-old man with generalized myasthenia gravis who was admitted to our hospital due to dyspnea. He developed severe respiratory distress in few hours and subsequently died of sudden cardiac death.
\end{abstract}

KEYWORDS: Myasthenia gravis; COVID-19; Sudden cardiac death

\section{ABBREVIATIONS}

MG: Myasthenia Gravis; SCD: Sudden Cardiac Death; COVID-19: Coronavirus Disease of 2019; SSRI: Selective Serotonin Reuptake Inhibitor; ABG: Arterial Blood Gas; ICU: Intensive Care Unit; RT-PCR: Reverse Transcription-Polymerase Chain Reaction; SARS-Cov2: Severe Acute Respiratory Syndrome Coronavirus 2; CT: Computed Tomography; LDH: Lactate Dehydrogenase; IVIG: Intravenous Gamma-Globulin; EKG: Electrocardiography.

\section{Vol No: 06, Issue: 02}

Received Date: June 02, 2021

Published Date: July 01, 2021

\section{*Corresponding Author}

Shekoofeh Yaghmaei

General practitioner, University of Fribourg, Av de Beaumont 5, Lausanne 1012, Fribourg,

Switzerland, Tel: +417826840 50;

E-mail: shekoofeh.yaghmaei@unifr.ch

Citation: Maysa Ghayyem, Sara Haseli, Shekoofeh Yaghmaei. (2021). COVID-19 and Sudden Cardiac Death in a Young Man with Myasthenia Gravis. Mathews J Case Rep. 6(2):68.

Copyright: Yaghmaei S, et al. (C) (2021). This is an open-access article distributed under the terms of the Creative Commons Attribution License, which permits unrestricted use, distribution, and reproduction in any medium, provided the original author and source are credited. 


\section{INTRODUCTION}

MG (Myasthenia Gravis) is a chronic autoimmune disorder caused by autoantibodies against the nicotinic acetylcholine receptors on the postsynaptic membrane at the neuro muscular junction. It is characterized by skeletal muscle weakness and fatigability, which typically increase with exercise [1]. Other commonly presented symptoms include diplopia, ptosis, and difficulty in swallowing. Approximately $20 \%$ of MG patients will experience myasthenia crisis, i.e., weakness of upper airway muscles leading to airway obstruction and aspiration. Myasthenic crisis usually happens within the first year of illness [2]. According to a study conducted by Liu C, et al. on 2195 myasthenic patients, the estimated mortality rate was $5.88 \%$ at a 10 -year followup. The most determinative death-related factors of MG are found out to be the severity of the disease at the beginning, the presence of acetylcholine receptor antibodies, and the occurrence of thymic pathology [3]. MG patients may also experience fluctuations in heart rate, which is mainly associated with autonomic dysfunction rather than the presence of cardiac conducting system pathology. Meanwhile, SCD (sudden cardiac death) has also been reported in some cases without a discovered definite connection [4].

Since the initial reports on the COVID-19 (coronavirus disease) cases in Wuhan, China, in December 2019, COVID-19 has rapidly become a global concern as it has spread all the world over. Nevertheless, little is known about its potential effect on MG patients. During this global pandemic, myasthenic patients are considered high risk not only due to the weakness of their respiratory muscles but also because of taking immunosuppressive medications [5].

The mortality rate of COVID-19 has been reported approximately $5.7 \%$ [6], which is primarily caused by pneumonia and acute respiratory distress [7]. Underlying diseases like high blood pressure, cardiovascular and cerebral disorders, diabetes, hyperlipidemia, and chronic renal failure increase mortality [8].

SCD has also been reported in the literature as an infrequent COVID-19-related death cause [9]. Prolongation of the QTc from the baseline average is mainly due to prescribed medications for COVID-19 patients, such as hydroxychloroquine and azithromycin [10]. Moreover, the viral infection itself can cause myocarditis and subsequently SCD.

Herein, we present a young man suffering from MG who experienced SCD following COVID-19.

\section{CASE PRESENTATION}

On April 3rd, 2020 a 37-year-old male was brought to the BuAli hospital, Tehran, Iran complaining about mild exertional dyspnea from six hours prior to his admission. He mentioned a background of generalized MG for the previous six years. His initial presenting symptoms were ptosis and weakness. As a result, he was treated with Pyridostigmine. The patient also stated a positive history of hypertension, Hashimoto thyroiditis, and depressive disorder, for which he has been receiving levothyroxine, losartan, and SSRI (selective serotonin reuptake inhibitor) respectively in the last six years.

The patient's vital signs upon his arrival are noted in table 1. Except for the mild crackles auscultated in the lower lobes of both lungs, all other physical examinations were normal. ABG (arterial blood gas) and laboratory blood tests were performed (The results are reported in Table 1 and Table 2). The Renal function test was normal, but an increase in liver enzymes was observed.

\begin{tabular}{|c|c|c|c|c|}
\hline \multicolumn{2}{|c|}{ Time after hospitalization } & Upon arrival & $8^{\text {th }}$ hour & $10^{\text {th hours }}$ \\
\hline \multicolumn{2}{|c|}{ Patient complaints } & $\begin{array}{l}\text { Mild exertional } \\
\text { dyspnea }\end{array}$ & Severe dyspnea & $\begin{array}{l}\text { Severe respiratory } \\
\text { distress, confusion }\end{array}$ \\
\hline \multirow{4}{*}{ Vital signs } & Temperature & $37.8^{\circ} \mathrm{C}$ orally & $37.6^{\circ} \mathrm{C}$ orally & $36.9^{\circ} \mathrm{C}$ orally \\
\hline & Pulse rate & $92 / \mathrm{min}$ & $105 / \mathrm{min}$ & $91 / \mathrm{min}$ \\
\hline & Respiratory rate & $22 / \mathrm{min}$ & $38 / \mathrm{min}$ & $45 / \mathrm{min}$ \\
\hline & Blood pressure & $140 / 90 \mathrm{mmHg}$ & $120 / 80 \mathrm{mmHg}$ & $100 / 60 \mathrm{mmHg}$ \\
\hline \multirow{5}{*}{ ABG Results } & PH & 7.41 & 7.31 & 7.32 \\
\hline & $\mathrm{PaO}_{2}$ & 64.7 & 94.4 & 57.7 \\
\hline & $\mathrm{PaCO}_{2}$ & 39.9 & 57.9 & 46.2 \\
\hline & $\mathrm{HCO}_{3}^{-}$ & 18 & 22 & 32 \\
\hline & Saturation & $\begin{array}{c}93.2 \% \\
\text { (In room air) }\end{array}$ & $\begin{array}{c}92.6 \% \\
\text { (5L/min } 02 \text { nasally) }\end{array}$ & $\begin{array}{c}95.2 \% \\
\text { (post intubation) }\end{array}$ \\
\hline
\end{tabular}

Table 1: Patient complaints, ABGs, and vital signs during the hospitalization course. 


\begin{tabular}{|c|c|c|c|c|c|c|c|c|c|c|c|c|c|c|}
\hline 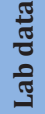 & D-Dimer & BUN & $\mathrm{Cr}$ & CRP & AST & ALT & LDH & $\mathrm{Pr}$ & TSH & T4 & T3 & WBC & HB & PLT \\
\hline$\ddot{g}$ & $\mathrm{ng} / \mathrm{ml}$ & $\mathrm{Mg} / \mathrm{dl}$ & $\begin{array}{c}\mathrm{Mg} / \\
\mathrm{dl}\end{array}$ & $\mathrm{Mg} / \mathrm{l}$ & $\mathrm{U} / \mathrm{l}$ & $\mathrm{U} / \mathrm{l}$ & $\mathrm{Au} / \mathrm{l}$ & $\mathrm{gr} / \mathrm{l}$ & $\mathrm{mU} / \mathrm{l}$ & $\begin{array}{c}\mathrm{ng} / \\
\mathrm{dl}\end{array}$ & $\mathrm{pg} / \mathrm{ml}$ & $/ \mu \mathrm{l}$ & $\mathrm{gr} / \mathrm{dl}$ & ML \\
\hline 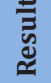 & 1460 & 18 & 0.8 & 65 & 46 & 37 & 430 & 5 & 3.1 & 1.71 & 3273 & $3580^{*}$ & 10.5 & $2.1 \times 10^{5}$ \\
\hline
\end{tabular}

Table 2: Results of the blood tests upon arrival. *With 89\% neutrophil. BUN: Blood urea nitrogen, Cr: Creatinine, CRP: C-reactive protein, AST: Aspartate aminotransferase, ALT: Alanine transaminase, LDH: Lactate dehydrogenase, Pr: Protein, TSH: Thyroid-stimulating hormone, T4: Thyroxine, T3: Triiodothyronine, WBC: White blood cell, HB: Hemoglobin, PLT: Platelet.

Due to patchy bilateral consolidation observed in the initial chest X-ray of the patient (Figure 1), he was transferred to ICU (intensive care unit). Upon ICU admission, Baseline ECG was within normal limits (Figure 2). In the following hours, dyspnea became gradually worse in so far as he developed with tachypnea and respiratory distress after 8 hours of his arrival. After applying 5 liters per minute 02 mask, another ABG was requested, which showed respiratory acidosis. As a result, endotracheal intubation was performed, and RT-PCR (reverse transcription-polymerase chain reaction) for SARSCov2 (Severeacute respiratory syndrome coronavirus 2) was requested.

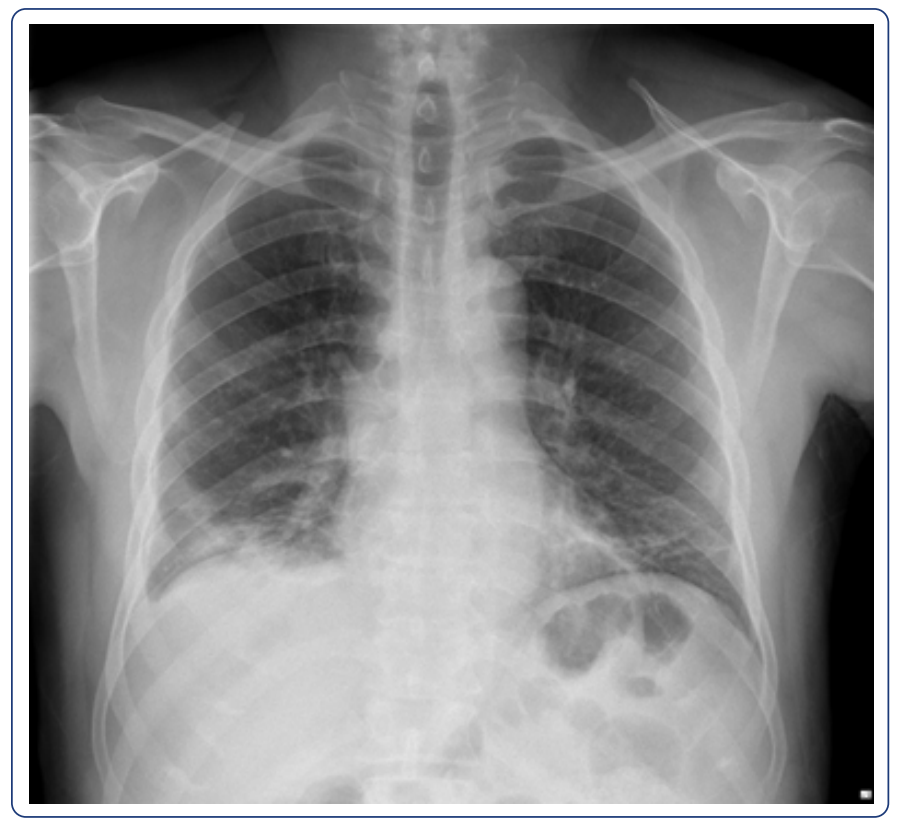

Figure 1: Initial admission posteroanterior chest radiography.

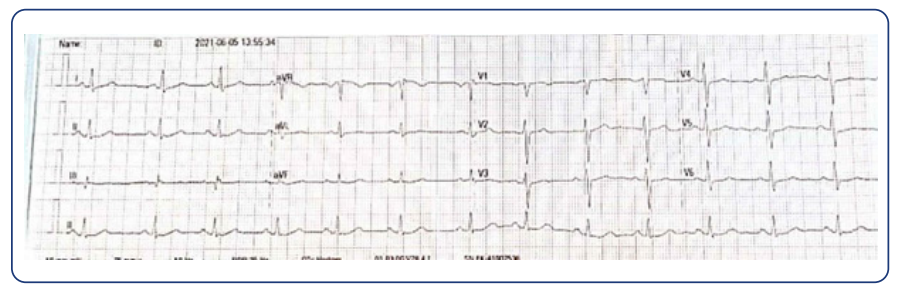

Figure 2: ECG showed normal sinus rhythm, normal axis, QTC intervals is $435 \mathrm{msec}$.
On the $9^{\text {th }}$ hour of admission, chest CT (Computed tomography) was performed, revealing bilateral multifocal ground-glassopacities with consolidation and mildright pleural effusion (Figure 3). Then, thoracocentesis has been performed for the patient, which results were as bellow: $\mathrm{LDH}$ (Lactate dehydrogenase) $=330 \mathrm{IU} / \mathrm{L}$ and protein $=4.6$ $\mathrm{g} / \mathrm{L}$ suggesting an exudative pleural fluid. We considered both infection and MG as the leading causes of the patient's respiratory failure, so he underwent plasmapheresis on the same day combined with $2 \mathrm{~g} / \mathrm{kg} /$ day IVIG (intravenous gamma-globulin). Moreover, he received Lopinavir/Ritonavir 400/100 mg and hydroxychloroquine 400mg in addition to the prophylactic anticoagulation. Echocardiography revealed normal cardiac function. Afterwards, the RT-PCR for SARSCov2 collected from the nasopharynx was reported positive. Despite all of these medical actions, no sign of recovery was depicted, and the patient's situation worsened as time was going on. Approximately 2 hours later, he suffered a cardiac arrest, and the cardiopulmonary resuscitation was unsuccessful, and he unfortunately died.

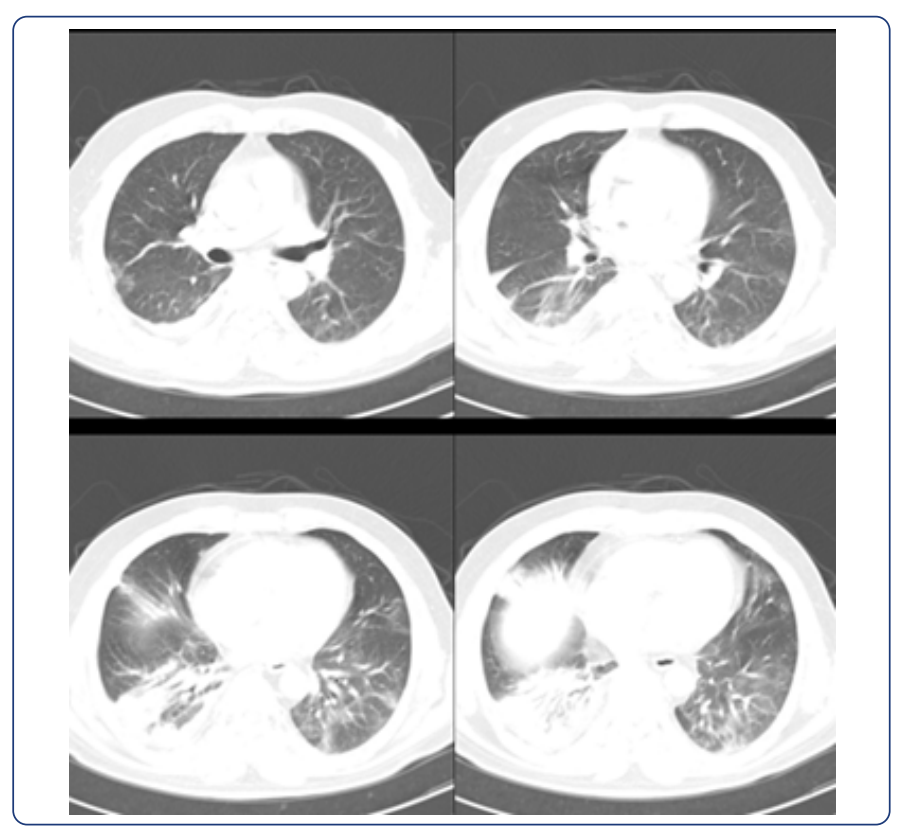

Figure 3: Chest spiral CT scan. 


\section{DISCUSSION}

MG is an immune-mediated disorder, most commonly affecting middle-aged females, but it is known to occur at any age [11]. It predominately involves the destruction of the neuromuscular junction by the acetylcholine receptorspecific antibodies. MG is diagnosed on the basis of clinical, electrophysiological, and serological findings [12]. Cardiac muscle is also a target for autoimmune inflammation in MG. Cardiac manifestations of MG include: heart failure, cardiomyopathy, pericarditis, and arrhythmia [4]. In addition, SCD in MG patients has been reported in literatures; however, no definite association has been established yet. Infection is considered a common trigger for MG exacerbation.

Despite the higher likelihood of COVID-19 severe disruptive effects on MG patients, due to receiving immunosuppressiveor immunomodulatory therapies and the weakness of respiratory muscles, in comparison with healthy population, no explicit association has been established, and this likelihood still remains debatable [13]. In addition to the infectious nature of COVID-19, its recommended therapies, such as azithromycin and hydroxychloroquine, may also lead to myasthenic exacerbation [14]. Most patients dealing with COVID-19 have a good prognosis, and a few patients (mostly elderly or people with comorbidities such as hypertension, diabetes, and chronic cardiac and renal disorders [15]) become critically ill. Although the COVID-19 mortality is mainly attributed to respiratory-related complications directly, cardiac consequences have recently been reported inthe literature [9]. According to a study conducted by Zhou et al. in Wuhan, China studying 191 COVID-19 patients, at least $17 \%$ of cases were found to have an elevated troponin level, and $23 \%$ were distinguished to experience heart failure [16]. Moreover, myocarditis, atrial and ventricular arrhythmias as well as cardiogenic shock have also been reported [17].

In the beginning, the symptoms of the presented patient were mild. Unfortunately, his condition worsened within a few hours. Finally, he developed severe respiratory distress requiring endotracheal intubation. In addition to $\mathrm{MG}$, the proarrhythmic effects of hydroxychloroquine which is prescribed for COVID-19, should also be considered as a possible cause of SCD. QT prolongation and torsades de pointes are common cardiac adverse effects of hydroxychloroquine [18]; nevertheless, the absolute risk of hydroxychloroquine causing SCD is minor and mainly observed among patients older than 60 years of age [19]. We did not have the ECG of the patient prior to his death, however, initial EKG (electrocardiography) was normal and did not show QT prolongation and torsades de pointes. In our case, myocarditis following viral infection could also be a cause of sudden cardiac death.

\section{CONCLUSION}

In conclusion, considering the disease process of the presented case and other similar reported cases [20], MG patients catching COVID-19 demonstrate different disease course compared with other COVID-19 cases. As a result, the unique evaluation and management for such patients seem necessary as MG patients are among the most vulnerable population, by means of explained throughout this report. It is also highly recommended to monitor cardiac condition in COVID-19 patients suffering from MG and admit them to ICU if possible. Moreover, repetition of blood gas appears necessary in order to forecast possible respiratory failure. Consultation with a cardiologist, EKG, echocardiography should be considered. Additionally, more studies are needed to make the disease process fully comprehensible. Reporting such cases can help the medical teams to be aware of the delicacy of treating COVID-19 patients with underlying diseases such as MG.

\section{CONSENT}

Written informed consent was obtained from the patient's family to publish this case report and accompanying images. A copy of the written consent is available for review by the Editor-in-Chief of this journal on request.

\section{ACKNOWLEDGMENTS}

We thank Ali Falsafi for his help in editing this report.

\section{AUTHORS CONTRIBUTION}

Maysa Ghayyem was the internist who has admitted the patient to the hospital and performed the treatment. Sara Haseli analyzed and interpreted the patient data regarding radiological findings, reviewed and edited the manuscript. Shekoofeh Yaghmaei prepared the draft of the manuscript and the tables. All authors read and proved the final manuscript.

\section{CONFLICT OF INTEREST}

None.

\section{REFERENCES}

1. Thanvi B, Lo T. (2004). Update on myasthenia gravis. Postgraduate medical journal. 80(950):690-700.

2. Juel VC, editor Myasthenia gravis: management of myasthenic crisis and perioperative care. Seminars in neurology; 2004: Copyright(C 2004 by Thieme Medical Publishers, Inc., 333 Seventh Avenue, New .... 
3. Liu C, Wang Q, Qiu Z, Lin J, Chen B, et al. (2017). Analysis of mortality and related factors in 2195 adult myasthenia gravis patients in a 10-year follow-up study. Neurology India. 65(3):518.

4. Shivamurthy P, Parker MW. (2014). Cardiac manifestations of myasthenia gravis: a systematic review. IJC Metabolic \& Endocrine. 5:3-6.

5. Ramaswamy SB, Govindarajan R. (2020). COVID-19 in refractory myasthenia gravis-a case report of successful outcome. Journal of neuromuscular diseases. mPreprint):1-4.

6. Baud D, Qi X, Nielsen-Saines K, Musso D, Pomar L, Favre G. (2020). Real estimates of mortality following COVID-19 infection. The Lancet infectious diseases. 20(7):773.

7. Vetter $\mathrm{P}, \mathrm{Vu}$ DL, L'Huillier AG, Schibler M, Kaiser L, Jacquerioz F. (2020). Clinical features of covid-19. British Medical Journal Publishing Group.

8. Shirazi S, Mami S, Mohtadi N, Ghaysouri A, Tavan H, et al. (2020). Sudden cardiac death in COVID-19 patients, a report of three cases. Future cardiology. 17(1):113-8.

9. Giudicessi JR, Roden DM, Wilde AA, Ackerman MJ. (2020). Genetic susceptibility for COVID-19-associated sudden cardiac death in African Americans. Heart rhythm.m17(9):1487-92.

10. Yadav R, Bansal R, Budakoty S, Barwad P. (2020). COVID-19 and sudden cardiac death: a new potential risk. Elsevier.

11. Aarli JA, ROMI F, Skeie GO, Gilhus NE. (2003). Myasthenia gravis in individuals over 40. Annals of the New York Academy of Sciences. 998(1):424-31.

12. Danikowski K, Jayaraman S, Prabhakar B. (2017). Regulatory $\mathrm{T}$ cells in multiple sclerosis and myasthenia gravis. Journal of neuroinflammation. 14(1):1-16.
13. Jacob S, Muppidi S, Guidon A, Guptill J, Hehir M, et al. (2020). Guidance for the management of myasthenia gravis (MG) and Lambert-Eaton myasthenic syndrome (LEMS) during the COVID-19 pandemic. Journal of the neurological sciences. 412 .

14. Anand P, Slama MC, Kaku M, Ong C, Cervantes-Arslanian AM, et al. (2020). COVID-19 in patients with myasthenia gravis. Muscle \& nerve. 62(2):254-8.

15. Ejaz H, Alsrhani A, Zafar A, Javed H, Junaid K, et al. (2020). COVID-19 and comorbidities: Deleterious impact on infected patients. Journal of Infection and Public Health.

16. Zhou F, Yu T, Du R, Fan G, Liu Y, et al. (2020). Clinical course and risk factors for mortality of adult inpatients with COVID-19 in Wuhan, China: a retrospective cohort study. The lancet. 395(10229):1054-62.

17. Kuck K-H. (2020). Arrhythmias and sudden cardiac death in the COVID-19 pandemic. Herz. 45(4):325-6.

18. Funck-Brentano C, Nguyen LS, Salem J-E. (2020). Retraction and republication: cardiac toxicity of hydroxychloroquine in COVID-19. The Lancet. 396(10245):e2-e3.

19. Simmering JE, Polgreen LA, Polgreen PM, Teske RE, Comellas AP, Carter BL. (2020). The cardiovascular effects of treatment with hydroxychloroquine and azithromycin. Pharmacotherapy: The Journal of Human Pharmacology and Drug Therapy. 40(9):978-83.

20. Hübers A, Lascano AM, Lalive PH. (2020). Management of patients with generalised myasthenia gravis and COVID-19: four case reports. Journal of Neurology, Neurosurgery \& Psychiatry. 91(10):1124-5. 